\title{
Intermédialités
}

Histoire et théorie des arts, des lettres et des techniques

Intermediality

History and Theory of the Arts, Literature and Technologies

\section{T’es où [Luc Courchesne]?}

\section{Luc Courchesne et Bernard Perron}

Numéro 9, printemps 2007

Jouer

Playing

URI : https://id.erudit.org/iderudit/1005535ar

DOI : https://doi.org/10.7202/1005535ar

Aller au sommaire du numéro

Éditeur(s)

Centre de recherche sur l'intermédialité

ISSN

1705-8546 (imprimé)

1920-3136 (numérique)

Découvrir la revue

Citer ce document

Courchesne, L. \& Perron, B. (2007). T’es où [Luc Courchesne]? Intermédialités /

Intermediality, (9), 157-175. https://doi.org/10.7202/1005535ar d'utilisation que vous pouvez consulter en ligne.

https://apropos.erudit.org/fr/usagers/politique-dutilisation/ 


\section{PORTRAIT}

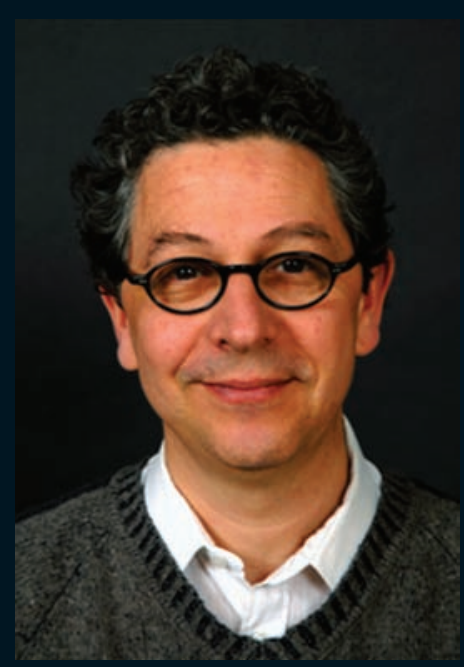

„Excusez-moi.»

„Puis-je vous poser une question?

»Allez-y toujours... Ça dépend quoi! « 


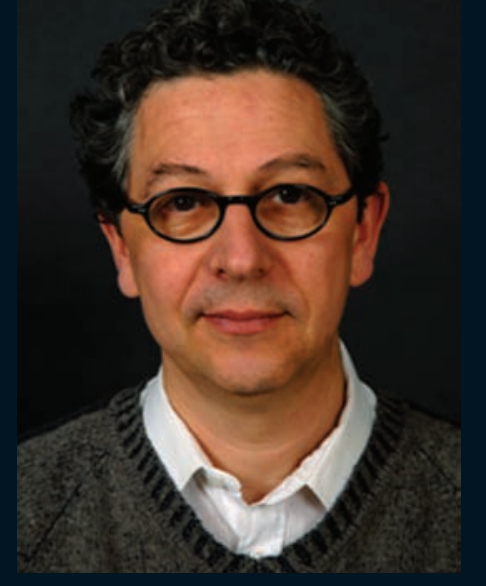

"Qui êtes-vous? «

»Je suis dans les arts médiatiques.» "Je suis inventif.«

")'ai 55 ans."

"Je suis un créateur-chercheur.»

»'enseigne le design à l'Université de Montréal.« 


\section{»Que faites-vous au juste?«}

»e suis collectionneur.«

»Je suis un portraitiste hypermédia.»

»'ai un penchant pour les paysages et les panoramas.«

»e conçois des outils et des contenus interactifs.«

\section{»Pouvez-vous en dire plus?«}

»Comme le dit Marie dans Portrait no. 1 (1990): «Le cinéma, c'est fascinant. Le spectateur est toujours là à épier tout ce qui se passe et personne dans le film ne s'en rend compte. Voir sans être vu. Du voyeurisme de première classe. Moi je vous regarde dans les yeux! D'Elastic Movies (1984) à Passages (1998), mes installations vidéo interactives ont souhaité interpeller le spectateur. C'est d'abord ce dernier que j'ai toujours voulu mettre en scène. Dans cette perspective, la conversation demeure à mon avis une situation toute naturelle pour entraîner le spectateur dans un échange virtuel. Bien que les questions et les réponses soient prédéterminées, les choix, eux, demeurent libres. Le spectateur doit pouvoir faire son propre cinéma.«

\section{»Cinéma...?«}

»Au-delà des discours utopistes de la fiction interactive qui prétendaient pouvoir lui offrir un récit qui s'adapterait vraiment à sa personnalité, le spectateur réalise en quelque sorte sa propre expérience. C'est pourquoi j'ai défini mon travail comme de «l'art expérienciel »'. Qu'il ait ou non le plein contrôle sur les images, c'est bien le spectateur qui, par son intervention, articule et "monte" les divers plans et séquences qu'il voit. $\mathrm{Si}$, comme le formule Lev Manovich dans The Language of New Media ${ }^{2}$, les rushes accumulés lors du tournage d'un film forment une base de données, le spectateur est amené à construire une expérience narrative à partir de cette dernière. Et dans la mesure où il y a une logique sousjacente à ce qui se déroule, un algorithme pour reprendre le terme de Manovich, l'expérience ne se résume pas à la simple consultation d'images.«

\section{»Continuez.«}

»e me sens toujours redevable des réalisateurs du cinéma expérimental qui ont remis en question et détourné le langage cinématographique. Mais peut-être autant, sinon plus, qu'un Michael Snow qui fixa sa caméra sur un singulier dispositif à bras mobile pour filmer sa Région centrale (1970-1971), j’ai dû - comme la plupart de mes camarades utilisant les nouvelles technologies de l'information et de la communication - créer mes propres outils d'expression. J'ai par exemple fait d'Hypercard mon principal instrument de création et de présentation pour toutes les installations vidéo interactives réalisées entre 1989 et 1998. «Système 
auteur, le premier du genre, mis sur le marché par Apple en juillet 1987, Hypercard permet à des non-programmeurs [comme moi] de concevoir et de réaliser des applications informatiques ${ }^{3}$ ». Grâce à ce logiciel et à la première génération de vidéodisques, j’ai pu rendre mes œuvres participatives.«

\section{»Êtes-vous allé plus loin?«}

»Mes premières œuvres demeurent des films interactifs, à savoir des œuvres dans lesquelles le spectateur peut intervenir pour orienter le déroulement du récit ou le défilement des séquences vidéo filmées. On le sait, le problème avec ce cinéma interactif - et ce qui a d'ailleurs mené à son échec ou à son insuccès populaire -, c'est que l'interactivité est nécessairement ponctuelle et lente. Le spectateur doit attendre le moment opportun pour « cliquer ». L'enchainement «entre l'actif et le passif, entre l'acteur et le spectateur » (Godard) ${ }^{4}$ ne peut dès lors être très fluide. Seule l'imagerie $3 \mathrm{D}$ en temps réel permet de résoudre cette impasse.«

\section{"La matrice de l'avenir?«}

»Devant la progression ahurissante des images de synthèse en temps réel et l'importance des jeux vidéo, la remédiatisation de séquences vidéo filmées ne saurait d'ailleurs être un critère essentiel pour parler de cinéma interactiff. Ainsi, puisque les choix de rendu $3 \mathrm{D}$ en temps réel se limitaient aux engins créés pour et réservés à des jeux comme Quake (jeu de tir à la première personne), ou encore à des plates-formes coûteuses et compliquées utilisées à des fins militaires ou aux travaux d'ingénierie exigeant la visualisation de données complexes, nous avons développé, mon équipe (Guillaume Langlois, Alexandru Catalin Duru) et moi un système de rendu «panoscopique» par l'entremise d'une interface de programmation pour la conception d'applications générant des images 3D. T'es où? (2005) n'est donc pas tant une œuvre qu'un logiciel de localisation de contenu, qu'un nouveau chantier d'exploration. Cela doit mener à une expérience de médiation loin d'être étrangère « à un parcours personnel entamé comme muséographe ${ }^{6} »$ «

\section{»Je ne vais pas au musée.»}

»Pourtant! Moi je m'y promènerais comme dans un parc. Par principe, je ne m'intéresserais à rien. J'attendrais d'être surpris par quelque chose. À l'occasion, une œuvre attirerait mon attention et j'aurais envie de me remettre au travail. M'écoutez-vous?《 


\section{PAYSAGE BY NUMBERS}

Une promenade, un parcours ludique

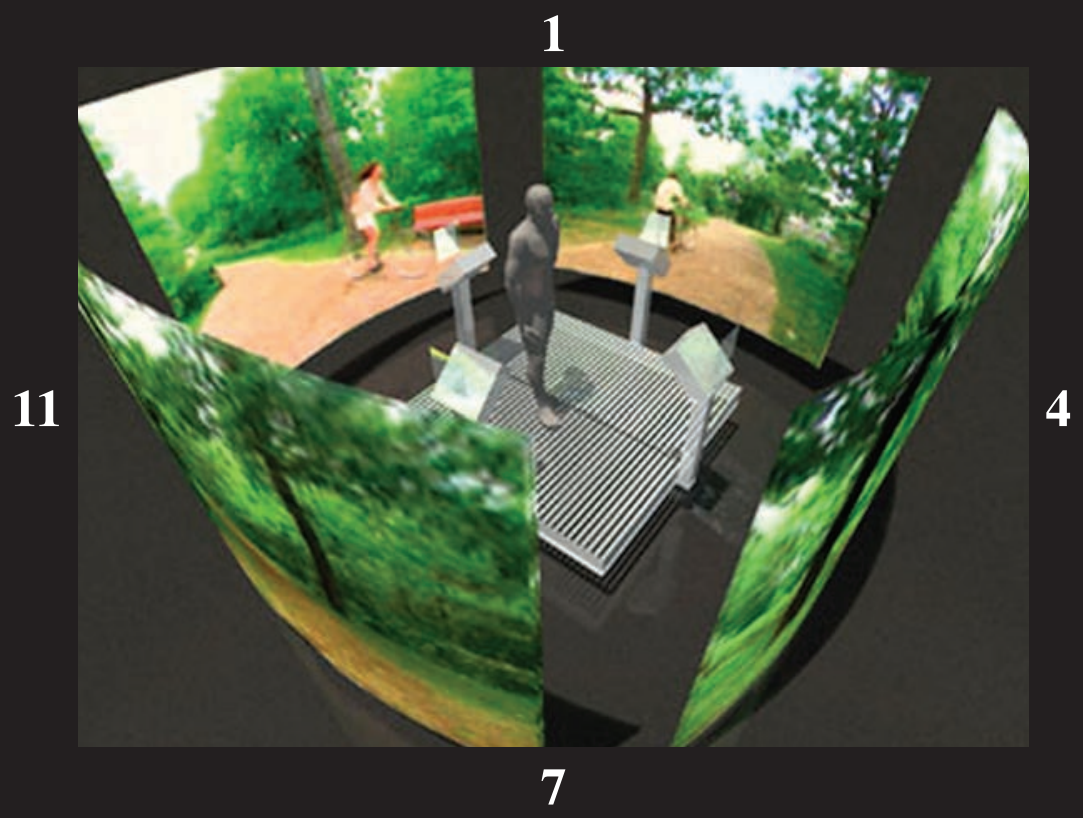


Une exploration par degrés, par niveaux

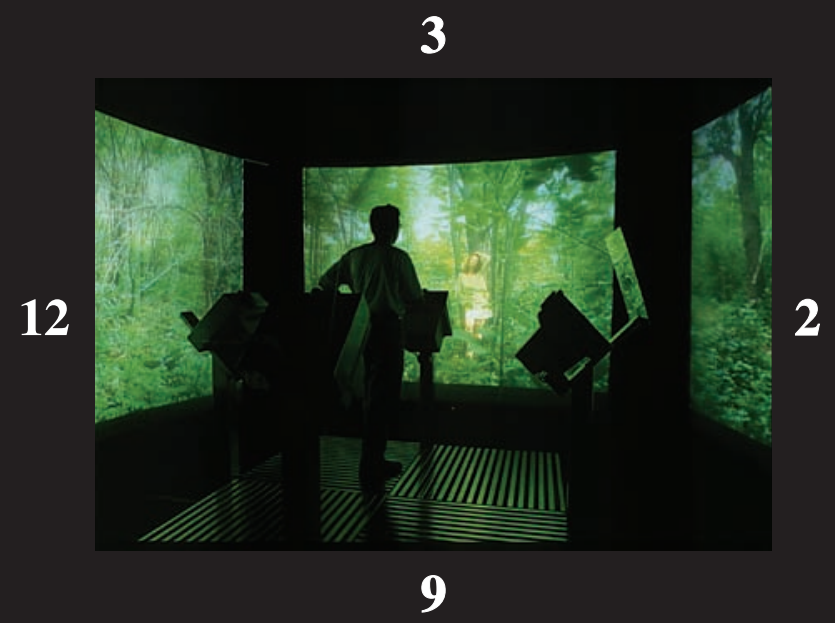


1. Tel que le notait Jean Gagnon, dès 1993, dans «Interactive Games and Dialogues »: "Luc Courchesne, a seasoned videomaker, was one of the first in Canada to turn interactive video (or hypermedia) to artistic ends. His aesthetic is based on play: his installations are set up in such a way that viewers, visitors, and players are confronted by a set of rules and objective parameters they can either accept or ignore - they play or they don't. Due to the gamelike nature of the work, the interaction is objective, and, as such, requires viewers to distance themselves to a certain degree; they must decide whether they want to play. Limits imposed by the technology constantly remind viewers that they are dealing with a technological go between that is itself part of the artist-engendered $\operatorname{game}^{7} »$.

4. Marie-Michèle Cron utilisait elle aussi cette analogie dans «Le monde virtuel de Luc Courchesne» : «[...] les espaces de conversation qu’il réalise attisent la réflexion sur une analyse des comportements des individus qui pousse à la fois à l'introspection et dans une sorte de griserie, car le spectateur est invité à parcourir un labyrinthe psychologique qui s'apparente à un jeu électronique émaillé de dangers et de récompenses, à décoder divers niveaux de langage à partir d'attitudes du quotidien, l'interactivité devenant ainsi source de métaphore de la rencontre et des ses règles. Car nos rapports avec la machine comme avec l'autre ne sont-ils pas souvent plus qu'autrement factices 9 ? »

7. Si le type d'interactivité créé par les arts médiatiques s'adresse en grande partie à un player, à un joueur-improvisateur qui va prendre en toute impunité ses décisions, cela ne désengage pas pour autant l'attitude plus agonistique d'un gamer.

Déjà parce qu'un bouton-poussoir permettait d'intervenir de manière plus directe - comparativement au capteur de mouvement infrarouge invisible - dans le défilement des images d'Encyclopédie clair-obscur (1987), l'œuvre a souvent été perçue comme «un genre de jeu d'arcade destiné à être brutalisé ${ }^{10} »$.

Lors des premières expositions de Portrait no. 1, plusieurs spectateursjoueurs se donnaient comme enjeu de converser le plus longtemps possible avec Marie avant qu'elle ne les éconduise. Certains d'entre eux soulignaient même la fin de leur rencontre en énonçant clairement leur game over.

Dans Paysage no. 1 (1997), le spectateur-joueur n'avait pas le choix de jouer un/leur jeu, puisqu'il devait attirer l'attention des personnages et les persuader de les conduire quelque part dans le parc du Mont-Royal.

Rien de surprenant alors que Luc Courchesne ait pris part à la création d'un jeu pour son Panoscope, jeu qui rend l'ergodicité plus intelligible que celle plus ouverte et moins réglé de T'es où?. Dans Catch \&un (2006), les joueurs incarnent un poulet, une vipère ou un renard qui se pourchassent 
les uns les autres. Le jeu fait revivre les vertiges des jeux de poursuite en s'aventurant cette fois sur des territoires artificiels non moins amusants que les arrière-cours de leur enfance.

11. Du spectateur qui joue (avec) les installations aux quatre personnages virtuels du théâtre vidéo interactif d'un Salon des ombres (1996) qui (se) jouent ensemble (du spectateur), en passant par l'artiste qui veut déjouer les attentes de celui qui s'aventure dans l'espace interactif, les œuvres de Luc Courchesne relèvent bien de ce que Jean-Louis Boissier a défini comme la «philosophie du jouable»: «Le jeu est protocole et dispositif, exercice et interprétation, distraction et travail. En parlant de jouable, on désigne une qualité, pas un type d'objets. On regarde comment des objets, des genres, des situations, des attitudes, des événements sont rendus jouables, par le numérique et l'interactivité 8 .» Le numérique se conjugue avec le ludique.

3. C'est le plan d'un homme s'avançant dans un long corridor, éclairé par des néons à distance régulière dont la lumière forme des arches, qui ponctue Elastic Movies, la première œuvre interactive de Luc Courchesne co-réalisée au Massachusetts Institute of Technology (MIT) en 1984. D'emblée, et sans compter la vue derrière l'épaule de l'homme qui fait référence à la perspective vidéoludique à la troisième personne, le spectateur-joueur est invité à faire le saut dans un autre univers. Come to our world. Press number 2.

2. Pour rendre plus personnelle la conversation avec les personnages de Portrait de famille (1993), Luc Courchesne a ajouté des « degrés d'intimité».

De questions plutôt banales d'ordre général (niveau 1), la discussion se tournera vers les activités, les motivations et/ou les croyances du personnage interrogé (niveau 2).

Suivant les (bonnes) réponses du spectateur-joueur, ce dernier pourra s'entretenir de sujets plus personnels et montrera ses sentiments (niveau 3).

Enfin, si les transitions ont été jusque-là bien accomplies, le personnage finira par confesser l'un de ses secrets (niveau 4). Cela mettra fin à la conversation ${ }^{11}$.

La conception du Salon des ombres (1996) n'est pas différente: «Il fallait construire l'histoire et son évolution dramatique. J'ai donc défini quatre niveaux: les personnages parlent entre eux; le visiteur entre en contact avec l'un des personnages; il est présenté aux trois autres; la discussion révèle aux personnages leur crise existentielle. [...] Le scénario est une sorte d'hyperarchitecture, permettant de loger chaque bribe du texte en fonction d'un développement. C'est un labyrinthe comparable à un réseau routier ${ }^{12}$.»

9. Parce qu'il veut faire du spectateur un visiteur, la logique de design interactif des installations de Luc Courchesne n'est pas très éloignée de celle du jeu 
vidéo. Elle repose aussi sur la conception et la réalisation de niveaux de jeu. Car il s'agit bien d'organiser la navigation par couches, de faire en sorte que ledit visiteur pénètre dans l'univers qui se présente à lui. D'abord conversationnelle, l'expérience relève de la navigation. Pour reprendre une expression anglaise utilisée par Henry Jenkins dans «Game Design as Narrative Architecture », l'expérience narrative tient à l'«environmental storytelling ${ }^{13}$ ». T'es où? participe du reality jam emblématique de notre temps. Il permet en cela de recadrer une idée de l'Encyclopédie clair-obscur, celle d'une cosmogonie navigable où le fil narratif est construit de façon spatiale.

12. T'es où? convie le spectateur-joueur à visiter un univers à plusieurs échelles. L'échelle o se présente sous la forme d'une trame ${ }_{3} \mathrm{D}$ navigable où le visiteur se familiarise avec l'espace qui l'entoure et les contrôles à sa disposition.

Le passage à l'échelle +1 fait apparaître des regroupements d'éléments visuels et sonores organisés comme des archives. Chacun des éléments de ces archives fait office de microportail. Ils sont les jalons d'une trame narrive qui se révèle selon le parcours et l'intérêt du visiteur.

Au passage à l'échelle +2 , les éléments de ces archives deviennent les particules de regroupements dont la composition générale rappelle les vues impressionnistes du monde à la fin du XIX ${ }^{\mathrm{e}}$ siècle et les premières formes de l'abstraction.

Le passage à l'échelle +3 révèle enfin un paysage de montagnes et de vallées évoquant l'idée du sublime pittoresque de la fin du XVIII ${ }^{\mathrm{e}}$ siècle.

À toutes les échelles de T'es où?, les visiteurs peuvent faire la rencontre d'autres promeneurs. Ultimement, c'est à un face-à-face avec lui-même que sera confronté le visiteur qui croisera le regard de son propre avatar au sein du monde artificiel qu'il arpente. 

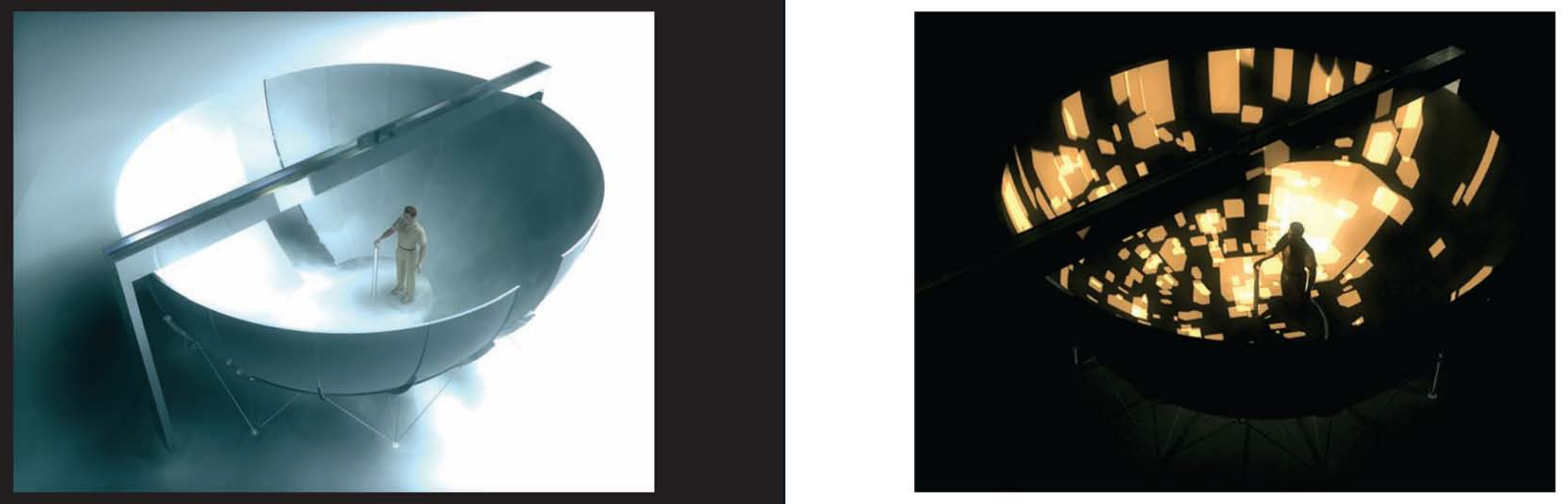

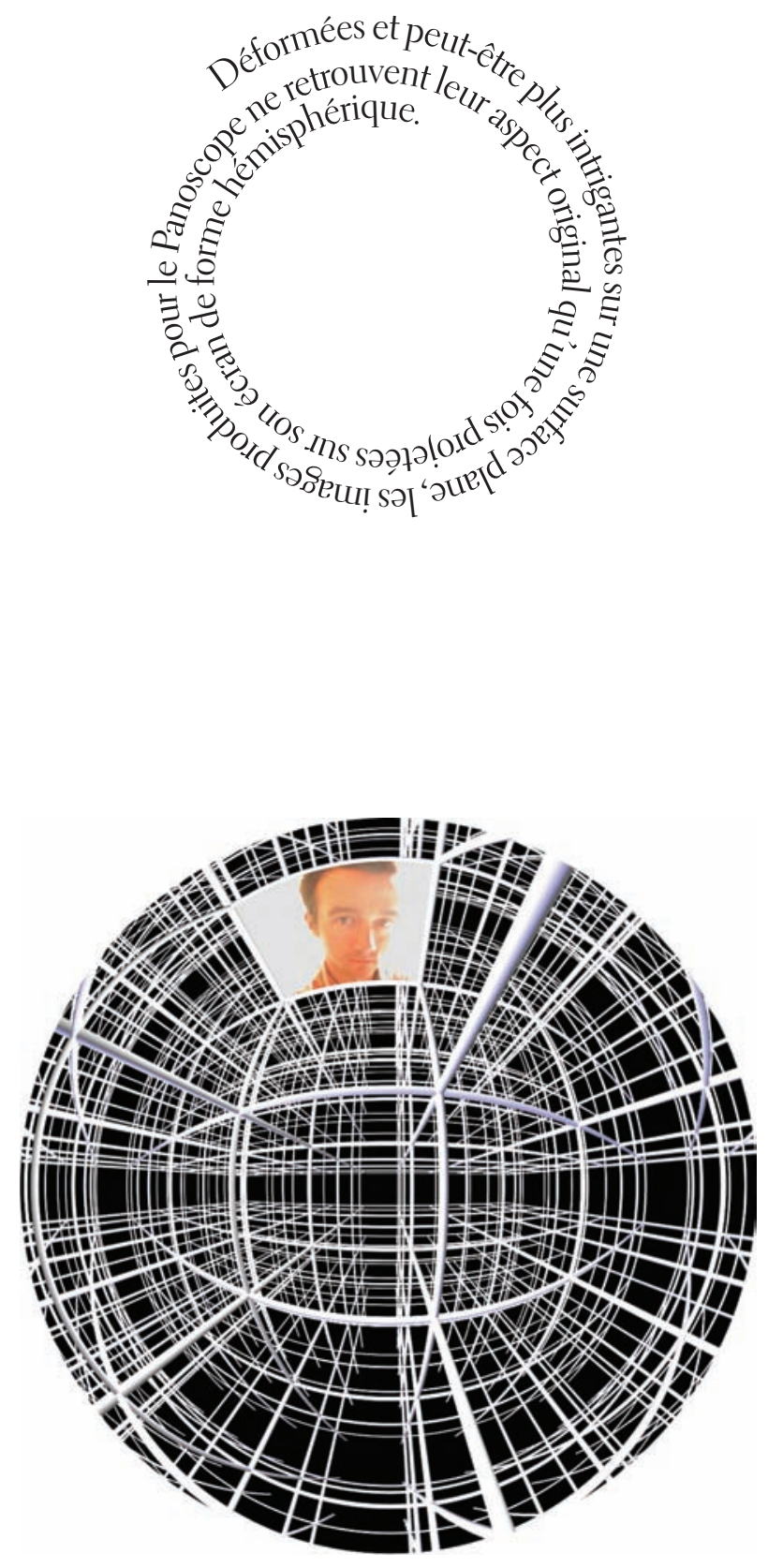

échelle o 
Avec son passage de la seule présentation de séquences vidéo préenregistrées à l'image $3 \mathrm{D}$ en temps réel, le Panoscope $360^{\circ}$ vise essentiellement à faciliter la création et la présentation de contenus interactifs et d'espaces navigables. Dans son grand format ( 5,5 mètres de diamètre sur 2,9 mètres de hauteur), le dispositif matérialise aussi, et peut-être surtout, les tenants de ce que l'on appelle l'immersion. Suivant la définition de Janet Murray: "Immersion is a metaphorical term derived from the physical experience of being submerged in water. We seek the same feeling from a psychologically immersive experience that we do from a plunge in the ocean or swimming pool: the sensation of being surrounded by a completely other reality, as different as water is from air, that takes over all of our attention, our whole perceptual apparatus ${ }^{14}$ ». Le visiteur est plongé dans l'image, dans la lumière.

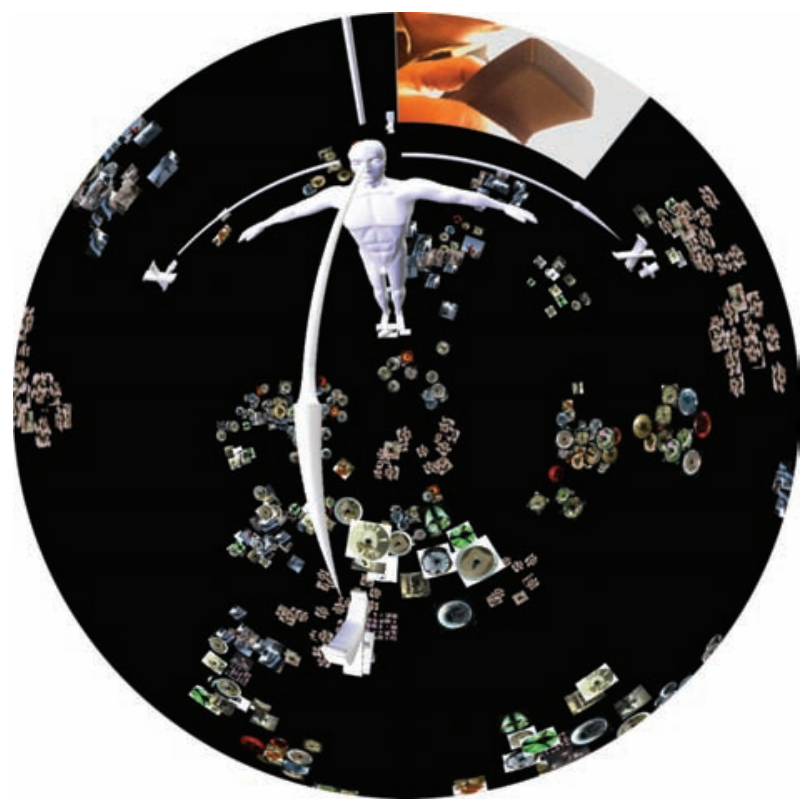

échelle +1 
Luc Courchesne affectionne particulièrement le moment où l'Alice de Lewis Carroll traverse de l'autre côté du miroir. Cette représentation définit à elle seule tout son travail. Certes, ce passage implique que le spectateur ne reste plus devant une image traditionnelle cadrée, mais plutôt qu'il plonge au cœur d'un univers virtuel. Ce n'est plus l'écran qui est panoramique (le widescreen), mais bien la perspective. Le cadre éclate. Le «monde du miroir » panoscopique n’est peutêtre pas inversé comme celui d'Alice, mais le dispositif, lui, l'est, en quelque sorte. En effet, le Panoscope a renversé le dôme plus connu du planétarium pour mieux ancrer le spectateur au centre de l'image et le faire pivoter. Dans cette caverne, les ombres sont projetées tout autour et proviennent du ciel (par monocanal, un seul projecteur).

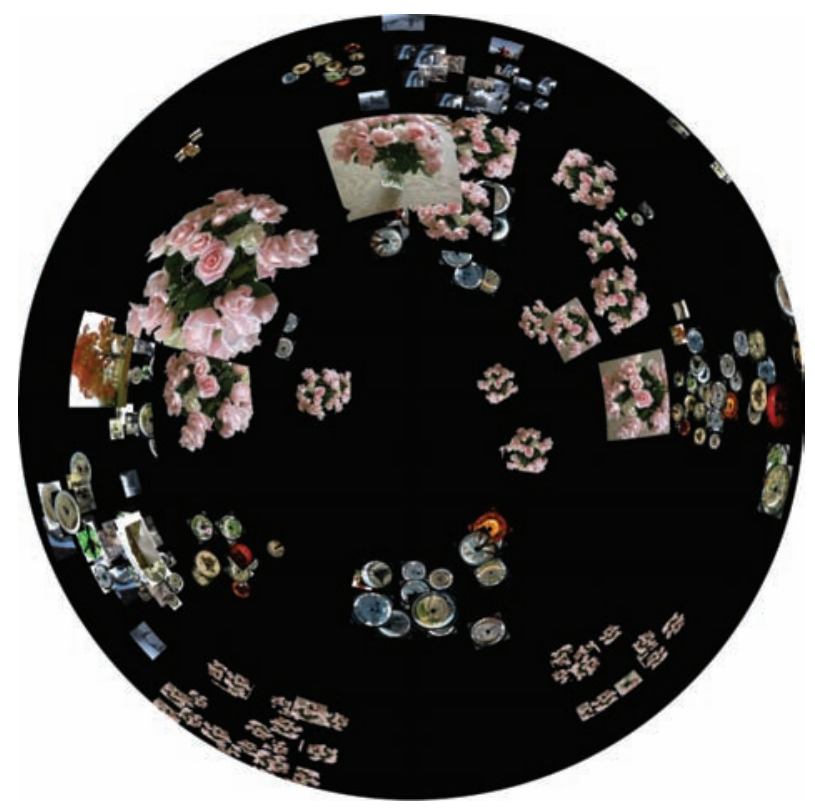

échelle +1 
Cela fait plus d'une décennie que Luc Courchesne s'affaire à et dans son Panoscope $360^{\circ}$. C'est son plus important projet de recherche, projet ayant d'ailleurs fait partie en 2005 du prestigieux WIRED NextFest : «an exposition that [brought] together innovations from top scientifists, researchers, and entrepreneurs. Created for the kid in all of us, WIRED NextFest 2005 [offered] more than 100 exhibits about the future of exploration, entertainment, transportation, health, communication, design, security, and imagination ${ }^{15} »$. Le Panoscope a été pressenti pour «vendre des voyages dans l'espace ». L'œuvre intitulée Vitesse de libération évoquera de façon poétique l'expérience de quitter la terre momentanément pour flotter en apesanteur au-dessus d'elle. Cette fois, le Panoscope sera inversé pour représenter l'infini de l'espace.

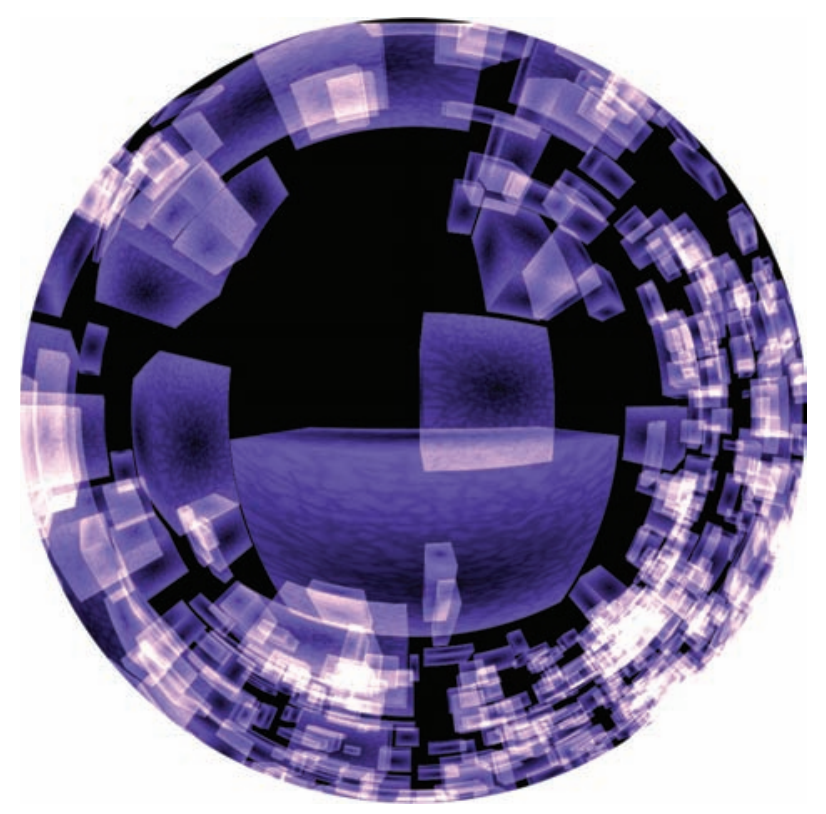

échelle +2 
Analysant les photographies du Journal panoscopique de Luc Courchesne, Brian Massumi explique que «la pupille ne fait pas partie de l'image en tant que telle; elle est son point aveugle formateur. Elle occupe la place de l'appareil muni d'une lentille panoramique et constitue un trou noir de la représentation, tenant lieu de son origine mécanique aussi bien que du corps de l'artiste éclipsé par la lentille à laquelle il doit l'image. » Se référant ensuite au Panoscope de petit format, Massumi note que «le spectateur entre dans le dispositif par ce qui, dans le disque photographique, est le trou noir où s'inscrivent l'origine humaine de l'image aussi bien que son processus mécanique et son principe monadique ${ }^{16}$ ». Cependant, le dispositif grand format fait disparaître le trou noir. Il le remplace par un joystick. À la lumière de ses propensions ludiques, c'est finalement au fameux cercle magique de Huizinga que nous renvoie le Panoscope $360^{\circ}$.

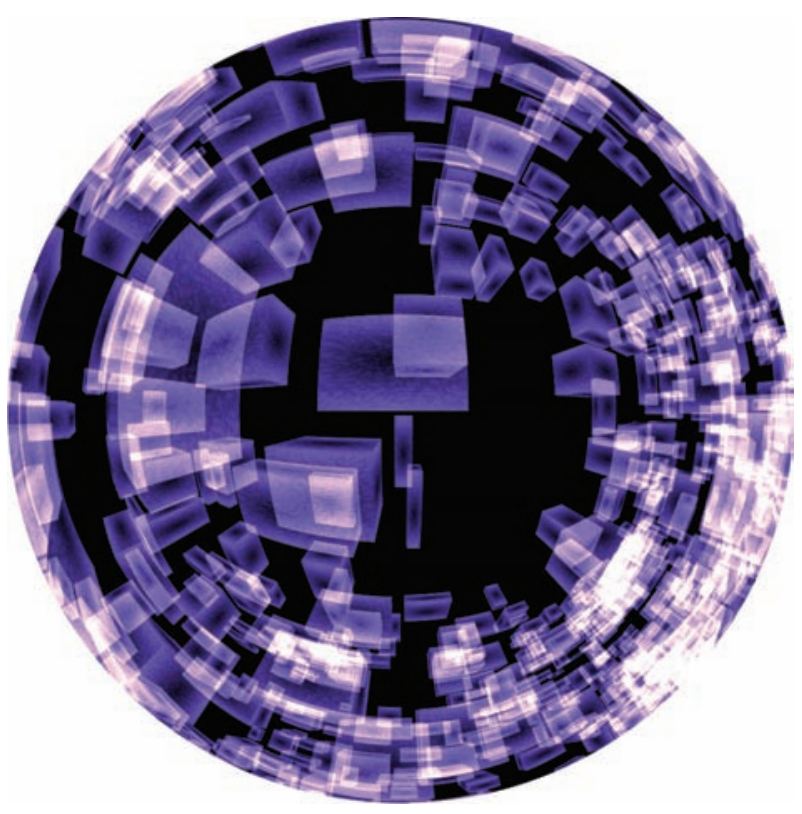

échelle +2 
En concevant T'es où?, Luc Courchesne a eu le sentiment de construire une sorte d'« Arche de Noé ». Parce que les bases technologiques n'ont cessé de s'effriter dans les années 1980 et 1990, il a alors été question de conserver les traces (et de perpétuer l'expérience) d'un monde voué à la disparition, puisque les systèmes qui le constituaient ont été rendus obsolètes par les avancées techniques. C'est pourquoi l'on retrouve des archives d'éléments visuels et sonores à l'échelle +1 de l'œuvre et que les images de roses - une figure qui a beaucoup marqué l'auteur - pullulent. Cela explique aussi que l'on rencontre, en plus des autres visiteurs filmés en direct à travers les liens de téléprésence, des interventions préenregistrées dans des fenêtres vidéo qui évoquent les portraits interactifs antérieurs.

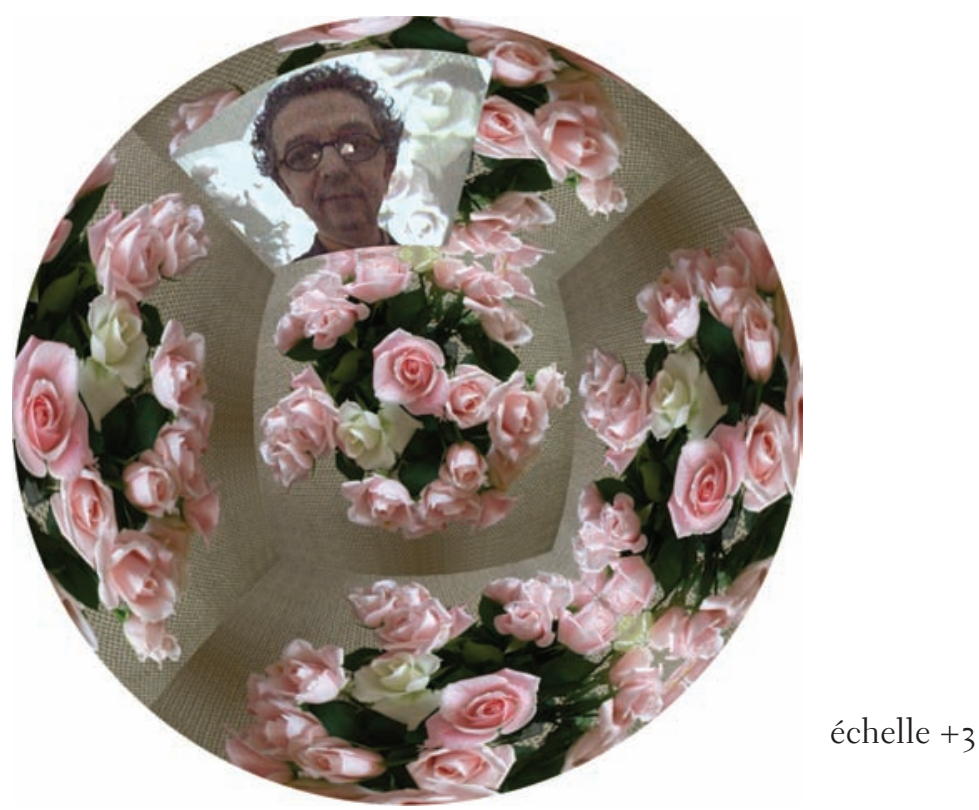



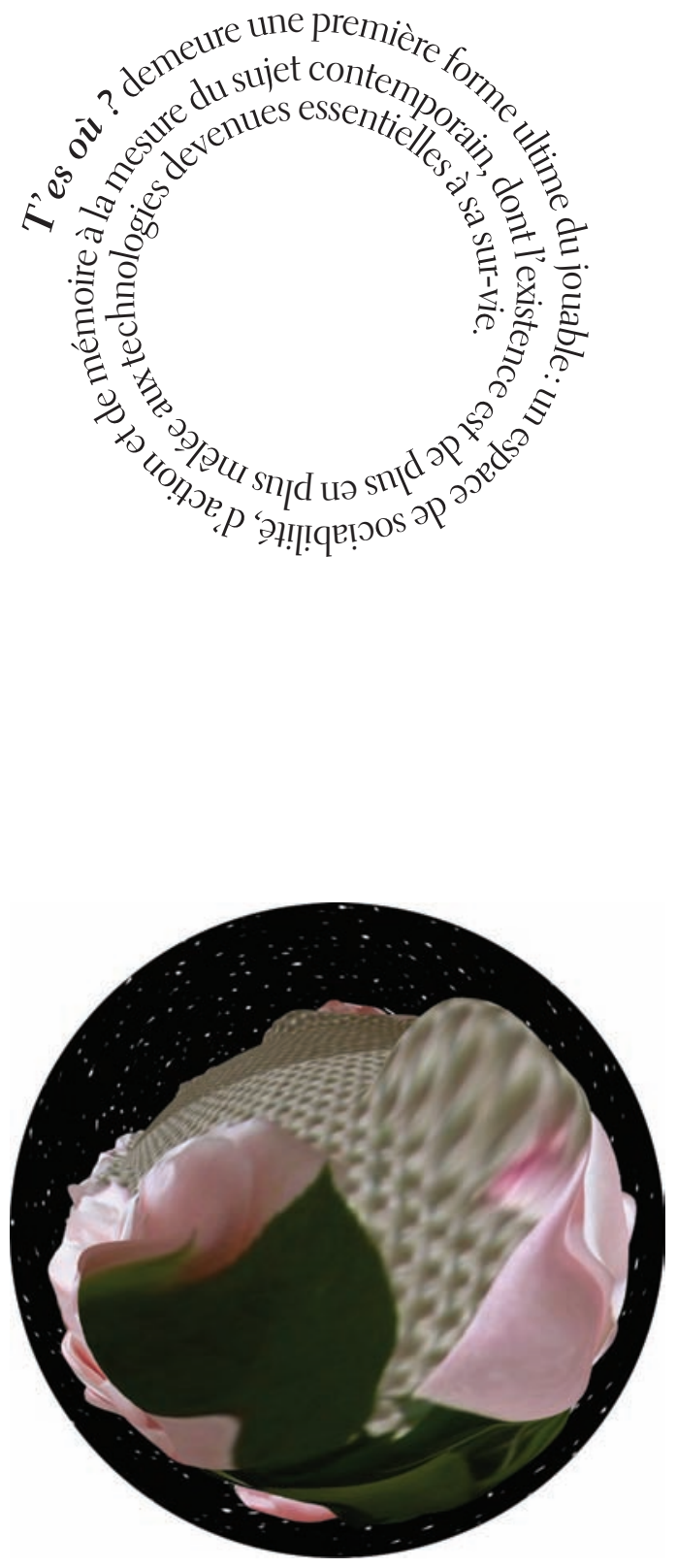

échelle +3 


\section{NOTES}

1. Luc Courchesne, "Art expérienciel: une étude de cas ", Éc/arts, n 3, février 2003, p. 186-197.

2. Lev Manovich, The Language of New Media, Cambridge, Massachusetts, The MIT Press, 2001, p. 236.

3. Luc Courchesne, «Art expérienciel: une étude de cas », p. 189.

4. Cité dans Erwan Higuinen, Charles Tesson, «Cinéphiles et ludophiles », Cahiers du cinéma, spécial "Jeux vidéo», hors-série, septembre 2002, p. 4.

5. Voir Bernard Perron, «Le cinéma interactif à portée de main », Alice Autelitano, Veronica Innocenti et Valentia Re (dirs.), The Five Senses of Cinema, Udine, Forum, 2005, p. 447-457.

6. Luc Courchesne, «Un créateur dans le musée-laboratoire: expérience de médiation », Musées/La lettre de l'OCIM, vol. 23, n 78, novembre-décembre 2001, p. 22.

7. Jean Gagnon, Catalogue Luc Courchesne: Interactive Portraits, National Gallery of Canada, Ottawa, du 13 novembre 1993 au 9 janvier 1994, p. 8.

8. Jean-Louis Boissier, «Philosophie du jouable. De la jouabilité », en ligne, http://www. jouable.net/philosophie_fr.html.

9. Marie-Michèle Cron, «Le monde virtuel de Luc Courchesne », La Presse, 24 décembre 1993, p. C8.

10. Luc Courchesne, «Art expérienciel: une étude de cas», p. 189.

11. Luc Courchesne, «Art expérienciel: une étude de cas», p. 192.

12. Dans Stéphanie Malphettes, "Vous n'êtes qu'une illusion », Le Monde, 30 décembre 1996, p. 34 .

13. Jenkins emprunte cette expression à Don Carson, qui a travaillé comme concepteur pour la division des parcs d'attraction de Walt Disney. Voir Henry Jenkins, «Games as Narrative Architecture», dans Pat Harrington, Noah Wardrip-Fruin (dirs.), First Person: New Media as Story, Performance, and Game, Cambridge, Massachusetts, The MIT Press, p. 121-123.

14. Janet Murray. Hamlet on the Holodeck. The Future of Narrative in Cyberspace, New York, The Free Press, 1997, p. 98.

15. Chris Anderson et Drew Schutte, «Welcome to the future», Catalogue WIRED NextFest.2005, Navy Pier, Chicago, du 24 au 26 juin, non paginé.

16. Brian Massumi. «Panoscopie. La photographie panoramique de Luc Courchesne», CV Photo, $\mathrm{n}^{\circ}$ 6o, avril 2003, p. 22-26. 